VOL. 50 (1994) [91-107]

\title{
THE SET OF IDEMPOTENTS OF A COMPLETELY REGULAR SEMIGROUP AS A BINARY ALGEBRA
}

\section{RAYMOND BROEKSTEEG}

\begin{abstract}
A semigroup $S$ is called $E$-solid if and only if for all idempotents $e, f, g \in S$ such that $e \mathcal{L} f \mathcal{R} g$ there exists an idempotent $h \in S$ such that $e \mathcal{R} h \mathcal{L} g$. Each completely regular semigroup is E-solid. We characterise the idempotents of an arbitrary E-solid regular semigroup as a set with a binary operation on it satisfying a given finite set of identities.
\end{abstract}

\section{INTRODUCTION}

The idempotents of a regular semigroup play an important role in determining the semigroup's structure. Indeed, many types of regular semigroups are (or can be) defined in terms of their idempotents alone. In $[9,10]$ Nambooripad introduced the concept of a biordered set and characterised the set of idempotents of a regular semigroup as a regular biordered set. Later on, Easdown [3] proved that biordered sets characterise idempotents of arbitrary semigroups.

An orthodox semigroup is a regular semigroup in which the product of two idempotents is again idempotent; because of this we can always restrict the binary operation of an orthodox semigroup to its set of idempotents to obtain a band. A semigroup $S$ is called $E$-solid if and only if for all idempotents $e, f, g \in S$ such that $e \mathcal{L} f \mathcal{R} g$ there exists an idempotent $h \in S$ such that $e \mathcal{R} h \mathcal{L} g$. An E-solid regular semigroup is a generalisation of an orthodox semigroup: each orthodox semigroup is E-solid but the product of two idempotents of an E-solid regular semigroup is not necessarily idempotent. By [4, Theorem 3] the idempotents of an E-solid regular semigroup generate a subsemigroup which is completely regular (that is, a union of groups). Thus the product of two idempotents of an E-solid regular semigroup $S$ is always contained in a (maximal) subgroup of $S$; because of this we can define a binary operation * on the set of idempotents of $S$ by defining, for each pair of idempotents $e, f \in S$,

$$
e * f=\text { the identity of the group } H_{e f}^{S} \text {. }
$$

Received 4th October, 1993

The author gratefully acknowledges the financial support of an Australian Postgraduate Research Award. The original idea of defining a binary operation on the set of idempotents of an E-solid regular semigroup comes from Dr. T.E. Hall, the author's supervisor, whom I thank for giving me his idea and for continually offering friendly advice about the presentation of this paper. I also thank Monica Mangold for finding a significant simplification of the proof of Theorem 6.2.

Copyright Clearance Centre, Inc. Serial-fee code: 0004-9729/94 \$A2.00+0.00. 
The resulting algebra $(E(S), *)$, which we call a solid binary algebra, generalises the band of idempotents of an orthodox semigroup: if $S$ is orthodox then $*$ is just the binary operation of $S$ restricted to $E(S)$. We show that solid binary algebras characterise the idempotents of E-solid regular semigroups.

\section{Summary}

In Section 3, we present Nambooripad's definition of a biordered set and we state some of his important results.

In Section 4, we define a solid binary algebra to be a nonempty set with a binary operation on it that satisfies a given finite set of identities. For an E-solid regular semigroup $S$, we show that the algebra $(E(S), *)$ as defined in the introduction, is in fact a solid binary algebra. We shall call $(E(S), *)$ the solid binary algebra determined by $S$. Conversely, we show that all solid binary algebras are determined by E-solid regular semigroups.

In Section 5, we show that morphisms between solid binary algebras are precisely the morphisms between E-solid regular semigroups with their domains restricted. For a solid binary algebra $(E(S), *)$ determined by an E-solid regular semigroup $S$, we show that the subalgebras of $(E(S), *)$ are determined by and are in a one to one correspondence with the idempotent generated regular subsemigroups of $S$. We also show that the solid binary algebra determined by the direct product of a family of Esolid regular semigroups is the direct product of the solid binary algebras determined by the members of the family.

In Section 6, we show that an algebra which is both a local semilattice algebra and a solid binary algebra is a normal band.

\section{Preliminaries}

For undefined notation and terms the reader is referred to $[2,5,6,10]$. As usual, for each semigroup $S$, we shall write $E(S)$ for the set of idempotents of $S$; by the core of $S$, we shall mean the subsemigroup generated by $E(S)$ in $S$; we shall say that a subset $A$ of $S$ is full if and only if $E(S) \subseteq A$.

Definition 3.1: (Nambooripad.) Consider any nonempty set $E$ and any partial binary operation o on $E$ with domain $D_{E}$ (if we write $e$ of for some $e, f \in E$, then 
it will be implicit that $\left.(e, f) \in D_{E}\right)$. Define

$$
\begin{aligned}
\boldsymbol{\omega}^{T} & =\{(e, f) \in E \times E \mid f \circ e=e\} \\
\omega^{l} & =\{(e, f) \in E \times E \mid e \circ f=e\} \\
\mathcal{R} & =\omega^{r} \cap\left(\omega^{r}\right)^{-1} \\
\mathcal{L} & =\omega^{l} \cap\left(\omega^{l}\right)^{-1} \\
\omega & =\omega^{r} \cap \omega^{l} .
\end{aligned}
$$

Also, for each pair of elements $e, f \in E$, define the sandwich set $S(e, f)$ of $e$ and $f$ to be the set

$$
\left\{h \in \omega^{l}(e) \cap \omega^{r}(f) \mid\left(\forall g \in \omega^{l}(e) \cap \omega^{r}(f)\right) e \circ g \omega^{r} e \circ h \text { and } g \circ f \omega^{l} h \circ f\right\} .
$$

Define $(E, \circ)$ to be a biordered set if and only if the following conditions and their duals hold:

(B1) $\omega^{r}$ and $\omega^{l}$ are quasi-orders on $E$ such that

$$
D_{E}=\left(\omega^{r} \cup \omega^{l}\right) \cup\left(\omega^{r} \cup \omega^{l}\right)^{-1}
$$

and for all $e, f, g \in E$

(B21) $f \in \omega^{r}(e) \Rightarrow f \mathcal{R} f \circ e \omega e$

(B22) $\left[g \omega^{l} f, f, g \in \omega^{r}(e)\right] \Rightarrow g \circ e \omega^{l} f \circ e$

(B31) $g \omega^{r} f \omega^{r} e \Rightarrow g \circ f=(g \circ e) \circ f$

(B32) $\left[g \omega^{l} f, f, g \in \omega^{r}(e)\right] \Rightarrow(f \circ g) \circ e=(f \circ e) \circ(g \circ e)$

(B4) $f, g \in \omega^{r}(e) \Rightarrow S(f, g) \circ e=S(f \circ e, g \circ e)$.

If $(E, 0)$ is a biordered set then define $(E, 0)$ to be regular if and only if $(\forall e, f \in E)$

(R) $S(e, f) \neq 0$.

Biordered sets arise naturally from semigroups: if $S$ is a semigroup with $E(S) \neq \emptyset$ and if we define

$$
D_{E(S)}=\{(e, f) \in E(S) \times E(S) \mid e f=e, e f=f, f e=f \text { or } f e=e\}
$$

then $E(S)$ together with the binary operation of $S$ restricted to $D_{E(S)}$ is a biordered set, which we shall denote by $(E(S), \circ)$. Easdown $[3]$ has proven conversely that every biordered set arises in this way from some semigroup. Nambooripad observed that if $S$ is a regular semigroup then $(E(S), 0)$ is a regular biordered set, and then found the following important converse [10, Theorem 5.2]: 
RESUlt 3.2. (Nambooripad.) For each regular biordered set $(E, 0)$ there is a fundamental regular semigroup, $T_{E}$, with $(E, \circ)$ as its biordered set of idempotents. Each fundamental regular semigroup whose biordered set of idempotents is isomorphic to $(E, \circ)$ is isomorphic to a full subsemigroup of $T_{E}$.

Definition 3.3: (Nambooripad.) Consider any biordered sets $(E, \circ)$ and $(F, \diamond)$; consider any mapping $\theta: E \rightarrow F$. Then $\theta$ is called a bimorphism if and only if $(\forall e, f \in E)$

$$
(e, f) \in D_{E} \Rightarrow(e \theta, f \theta) \in D_{F} \text { and }(e \circ f) \theta=e \theta \diamond f \theta \text {. }
$$

Furthermore, $\theta$ is called a regular bimorphism if and only if $(\forall e, f \in E)$

(a) $S_{E}(e, f) \theta \subseteq S_{F}(e \theta, f \theta)$

(b) $S_{E}(e, f) \neq \emptyset \Leftrightarrow S_{F}(e \theta, f \theta) \neq \emptyset$.

An isomorphism between biordered sets is a bijective bimorphism whose inverse is also a bimorphism.

DeFinition 3.4: (Nambooripad.) Consider any biordered sets $(E, \circ)$ and $(F, \diamond)$. Then $(F, \diamond)$ is a biordered subset of $(E, 0)$ if and only if

(a) $F \subseteq E$

(b) $D_{F}=D_{E} \cap(F \times F)$

(c) $\diamond=$ o $D_{F}$.

Furthermore, when $(F, \diamond)$ is a biordered subset of $(E, 0)$, we say that $(F, \diamond)$ is relatively regular in $(E, \circ)$ if and only if the inclusion mapping $1_{F E}: F \hookrightarrow E$ is a regular bimorphism.

Note that a relatively regular biordered subset of a regular biordered set is necessarily regular.

In Section 5, the direct product of a family of biordered sets is used; because the direct product of a family of biordered sets is not defined in [10], we define it here.

DEFinition 3.5: We define the direct product $\prod_{i \in I}\left(E_{i}, \circ_{i}\right)$ of a nonempty family $\left\{\left(E_{i}, o_{i}\right) \mid i \in I\right\}$ of biordered sets in the following way. The set $\prod_{i \in I} E_{i}$ has componentwise multiplication . with domain

$$
\left\{(e, f) \in \prod_{i \in I} E_{i} \times \prod_{i \in I} E_{i} \mid(\forall i \in I)\left(e_{i}, f_{i}\right) \in D_{E_{i}}\right\}
$$

Restricting the domain of - further to the set

$$
\left\{(e, f) \in \prod_{i \in I} E_{i} \times \prod_{i \in I} E_{i} \mid e \cdot f=e, e \cdot f=f, f \cdot e=f \text { or } f \cdot e=e\right\}
$$


yields a (partial) multiplication $\circ$ on $\prod_{i \in I} E_{i}$. We call $\left(\prod_{i \in I} E_{i}, \circ\right)$ the (biordered set) direct product of $\left\{\left(E_{i}, \circ_{i}\right) \mid i \in I\right\}$.

The next two results follow easily from the definition of the direct product and no proof will be given for them.

RESUlT 3.6. The direct product $\prod_{i \in I}\left(E_{i}, \circ_{i}\right)$ of a nonempty family $\left\{\left(E_{i}, \circ_{i}\right) \mid i \in I\right\}$ of biordered sets is a biordered set such that, for each pair of elements $e, f \in \prod_{i \in I} E_{i}$,

$$
S_{\prod_{i}}(e, f)=\prod_{i \in I} S_{E_{i}}\left(e_{i}, f_{i}\right)
$$

RESULT 3.7. The biordered set of idempotents of the direct product $\prod_{i \in I} S_{i}$ of a nonempty family $\left\{S_{i} \mid i \in I\right\}$ of semigroups (such that $E\left(S_{i}\right) \neq \emptyset$ for each $i \in I$ ) is the direct product $\prod_{i \in I}\left(E\left(S_{i}\right), \circ_{i}\right)$ of the family $\left\{\left(E\left(S_{i}\right), \circ_{i}\right) \mid i \in I\right\}$ of biordered sets of the members of $\left\{S_{i} \mid i \in I\right\}$.

Two important types of regular biordered sets are solid regular biordered sets and local semilattices: a solid regular biordered set is a regular biordered set in which the relations $\mathcal{L}$ and $\mathcal{R}$ commute, that is,

$$
\mathcal{L} \circ \mathcal{R}=\mathcal{R} \circ \mathcal{L}
$$

a local semilattice is a (regular) biordered set in which every sandwich set contains exactly one element.

\section{Solid binary algebras}

Definition 4.1: Consider any nonempty set $E$ and any binary operation * : $E \times E \rightarrow E$. Define $(E, *)$ to be a solid binary algebra if and only if $(E, *)$ satisfies the following identities and their duals:

(SBA1) $e * e=e$

(SBA2) $e *((e * f) * g)=(e * f) * g$

(SBA3) $\quad(e * f) *(f * e)=e *(f * e)$

(SBA4) $(e * f) *(e *(f * g))=e *(f * g)$

(SBA5) $\quad((e * f) * e) *(e * g)=(e * f) *(e * g)$

(SBA6) $\quad((e * f) *(e * g)) * e=((e * f) * e) *((e * g) * e)$.

LEMмA 4.2. Consider any solid binary algebra $(E, *)$. Then $(E, *)$ satisfies the following two identities and their duals:

(SBA7) $e *(e * f)=e * f$

(SBA8) $(e * f) * e=e *(f * e)$. 
Proof: We easily see that (SBA7) holds by using (SBA1) and (SBA2). Note that the dual of (SBA3) is

$$
(e * f) *(f * e)=(e * f) * e,
$$

and so (SBA3) and its dual gives (SBA8). The duals of (SBA7) and (SBA8) follow dually (in fact (SBA8) is self dual).

REMARK 4.3. The author has a direct proof from the axioms that the subalgebra generated by any pair of elements in a solid binary algebra is a band, but since the proof is long, the proof will not be given here; instead, using a structure theorem for solid binary algebras, the author will give a (shorter) proof in a future paper [1]. Thus the free solid binary algebra on two generators is just a free band with six elements. It is interesting to note that the free local semilattice algebra on two generators has an infinite number of elements: see $[7,8]$.

We now show that each E-solid regular semigroup determines a solid binary algebra. First note that by [4, Theorem 3], we have that the product of any two idempotents in an E-solid regular semigroup $S$ is always contained in a subgroup of $S$. Now as in [12, Section 3], for each pair of idempotents $e, f$ in an E-solid regular semigroup, we shall write $(e f)^{-1}$ for the inverse of $e f$ in the group $H_{e f}$.

Theorem 4.4. Consider any $E$-solid regular semigroup $S$ and let $(E, \circ)$ be the biordered set of idempotents of $S$. Define * :E $\times E \rightarrow E$ by $(\forall e, f \in E)$

$$
e * f=\text { the identity of the group } H_{e f}^{S} \text {. }
$$

Then $(E, *)$ is a solid binary algebra such that $(\forall e, f \in E)$

(a) $(\forall g \in S(e, f)) e \circ g \mathcal{R} e * f \mathcal{L} g \circ f$

(b) $e * f=(e f)(e f)^{-1}=(e f)^{-1}(e f)=e(f e)^{-1} f \in S(f, e)$

(c) (i) $e * f=f$ if and only if $e f=f$

(ii) $e * f=e$ if and only if $e f=e$

(d) the restriction of $*$ to the domain of o coincides with $\circ$, that is,

$$
\circ=* \mid D_{E}
$$

Proof: We shall first prove (a), (b), (c) and (d). Consider any $e, f \in E$ and any $g \in S(e, f)$. By [10, Theorem 1.1] we have that eg $\mathcal{R}$ ef $\mathcal{L} g f$. Now since $e f \mathcal{H} e * f$, it is true that eg $\mathcal{R} e * f \mathcal{L} g f$. But $g \in \omega^{l}(e) \cap \omega^{r}(f)$ and so $e g=e \circ g$ and $g f=g \circ f$. Thus

$$
e \circ g \mathcal{R} e * f \mathcal{L} g \circ f
$$


This proves (a). By the definition of $(e f)^{-1}$, we have that $e f(e f)^{-1}=(e f)^{-1} e f$ is the identity element of the group $H_{e f}$, and so $e * f=e f(e f)^{-1}=(e f)^{-1} e f$. Since $e(e f)^{-1}=(e f)^{-1}$ and $(f e)^{-1}=(f e)^{-1} e$, we have that

$$
\begin{aligned}
e f(e f)^{-1} & =e f e(e f)^{-1} \\
& =e(f e)^{-1} f e f e(e f)^{-1} \\
& =e(f e)^{-1} \text { efef }(e f)^{-1} \\
& =e(f e)^{-1} e f \\
& =e(f e)^{-1} f .
\end{aligned}
$$

Finally, since $(f e)^{-1} \in V(f e)$, we have that $e(f e)^{-1} f \in S(f, e)$. This proves (b). Now suppose that $e * f=f$. Then $f=e f(e f)^{-1}$ and so $e f=e\left(e f(e f)^{-1}\right)=e f(e f)^{-1}=f$. Conversely, suppose that $e f=f$. Then $e * f=e f(e f)^{-1}=f f^{-1}=f f=f$. This proves (c)(i), and (c)(ii) follows dually. To show that $o=* \mid D_{E}$, suppose that $(e, f) \in D_{E}$, the domain of $o$. Then ef is idempotent and so ef is the identity of $B_{e f}$. Hence $e * f=e f=e \circ f$. This proves (d). We now show that $(E, *)$ is a solid binary algebra. Consider any $e, f, g \in E$. It is easy to see that (SBA1) holds. Since $(e * f) * g=e f(e f)^{-1} g\left(e f(e f)^{-1} g\right)^{-1}$, we have that $e((e * f) * g)=(e * f) * g$ and so by $(c)(i)$, we have that

$$
e *((e * f) * g)=(e * f) * g .
$$

Hence (SBA2) holds. Now note that

$$
\begin{aligned}
e f(e f)^{-1} f e(f e)^{-1} & =e f(e f)^{-1} e f(f e)^{-1} \\
& =e f(f e)^{-1} \\
& =e(f e)^{-1} \mathcal{H} e f e(f e)^{-1}
\end{aligned}
$$

and since $e f e(f e)^{-1}$ is idempotent, we have that $e *(f * e)=e f e(f e)^{-1}$. It follows that

$$
(e * f) *(f * e)=e f e(f e)^{-1}=e *(f * e) .
$$

Hence (SBA3) holds. Since $e * f=e f(e f)^{-1}$ and $e *(f * g) \mathcal{H} e f g(f g)^{-1}$, we have that $(e * f)(e *(f * g))=e *(f * g)$. Thus by $(\mathrm{c})(\mathrm{i})$,

$$
(e * f) *(e *(f * g))=e *(f * g) \text {. }
$$

Thus (SBA4) holds. Also

$$
\begin{aligned}
(e * f)(e * g) & =e f(e f)^{-1} e g(e g)^{-1} \\
& =e f(e f)^{-1} e e g(e g)^{-1} \mathcal{H}((e * f) * e) *(e * g)
\end{aligned}
$$


and so

$$
((e * f) * e) *(e * g)=(e * f) *(e * g) .
$$

Hence (SBA5) holds. Now note that

$$
\begin{gathered}
e f(e f)^{-1} e g(e g)^{-1} \mathcal{R} e f(e f)^{-1} e g(e g)^{-1} e \\
\text { and } \quad(e * f) *(e * g) \mathcal{H}(e * f)(e * g)=e f(e f)^{-1} e g(e g)^{-1} .
\end{gathered}
$$

Hence, by Green's Lemma,

$$
((e * f) *(e * g)) e \mathcal{H} e f(e f)^{-1} e g(e g)^{-1} e
$$

and so

$$
((e * f) *(e * g)) * e \mathcal{H} e f(e f)^{-1} e g(e g)^{-1} e .
$$

Also

$$
((e * f) * e) *((e * g) * e)=\left(e f(e f)^{-1} e\right) *\left(e g(e g)^{-1} e\right) \mathcal{H} e f(e f)^{-1} e g(e g)^{-1} e
$$

Thus

$$
((e * f) *(e * g)) * e=((e * f) * e) *((e * g) * e) .
$$

Hence (SBA6) holds. Dually, $(E, *)$ satisfies the duals of the above identities, which completes the proof.

REMARK 4.5. We call $(E, *)$ the solid binary algebra determined by $S$.

THEOREM 4.6. For each solid regular biordered set $(E, \circ)$ there is a unique binary operation $*: E \times E \rightarrow E$ such that

(a) $(E, *)$ is a solid binary algebra

(b) $\circ$ is $*$ restricted to the domain

$$
D_{E}^{*}=\{(e, f) \in E \times E \mid e * f=e, e * f=f, f * e=f \text { or } f * e=e\} .
$$

Proof: Consider any solid regular biordered set $(E, 0)$ and find an E-solid regular semigroup $S$ whose biordered set of idempotents is $(E, \circ)$ (for example $T_{E}$ ). Define $*: E \times E \rightarrow E$ by $(\forall e, f \in E)$

$$
e * f=\text { the identity of the group } H_{e f}^{S} .
$$

Using Theorem 4.4, we can easily see that (a) and (b) hold. We now show that $*$ is uniquely determined by (a) and (b). Consider any binary operation $+: E \times E \rightarrow E$ that satisfies (a) and (b). Consider any $e, f \in E$. We first show that $e *(f * e) \mathcal{R} e+(f+e)$. Since $*$ and + agree on the domain of $o$, we have that

$$
e+(f+e)=e *(f+e) \text { and } f+e=f+(f+e)=f *(f+e) .
$$


Hence $e+(f+e)=e *(f *(f+e))$. Now using (SBA8), (SBA5) and then (SBA4), we have that

$$
\begin{aligned}
(e *(f * e)) *(e+(f+e)) & =((e * f) * e) *(e *(f *(f+e))) \\
& =(e * f) *(e *(f *(f+e))) \\
& =e *(f *(f+e)) \\
& =e+(f+e) .
\end{aligned}
$$

By symmetry, we have that $(e+(f+e))+(e *(f * e))=e *(f * e)$. Thus

$$
e *(f * e) \mathcal{R} e+(f+e) \text {. }
$$

We now show that $e * f \mathcal{R} e+f$. By (SBA7) we have that $e+f \in \omega^{r}(e)$, and so by (B21), we have that

$$
e+f \mathcal{R}(e+f)+e .
$$

By (SBA8) we have that $(e+f)+e=e+(f+e)$, and so

$$
e+f \mathcal{R} e+(f+e) \text {. }
$$

Also, by symmetry, we have that $e * f \mathcal{R} e *(f * e)$. We now have that

$$
e * f \mathcal{R} e *(f * e) \mathcal{R} e+(f+e) \mathcal{R} e+f .
$$

Dually, we have that $e * f \mathcal{L} e+f$. It follows that $e * f=e+f$. This completes the proof.

\section{REMARK 4.7.}

(a) We call $(E, *)$ the solid binary algebra determined by $(E, o)$.

(b) By Theorem 4.4, we have that $(\forall e, f \in E)$

(i) $(\forall g \in S(e, f)) e \circ g \mathcal{R} e * f \mathcal{L} g \circ f$

(ii) $e * f \in S(f, e)$

(c) Notice that each E-solid regular semigroup with $(E, \circ)$ as its biordered set of idempotents determines $(E, *)$ in the sense of Theorem 4.4.

THEOREM 4.8. Each solid binary algebra is determined by a unique solid regular biordered set.

Proof: Consider any solid binary algebra $(E, *)$. Let

$$
\begin{aligned}
\omega^{r} & =\{(e, f) \in E \times E \mid f * e=e\} \\
\omega^{l} & =\{(e, f) \in E \times E \mid e * f=e\} \\
D_{E} & =\left(\omega^{r} \cup \omega^{l}\right) \cup\left(\omega^{r} \cup \omega^{l}\right)^{-1} \\
\circ & =* \mid D_{E} .
\end{aligned}
$$


We show that $(E, \circ)$ is a solid regular biordered set. Then, clearly, it is the only solid regular biordered set that determines $(E, *)$ in the sense of Theorem 4.6. We can easily see that $\omega^{r}$ and $\omega^{l}$ are quasi-orders by using identities (SBA1), (SBA2) and the dual of (SBA2). Now note that

and

$$
\begin{aligned}
\omega^{r} & =\{(e, f) \in E \times E \mid f \circ e=e\} \\
\omega^{l} & =\{(e, f) \in E \times E \mid e \circ f=e\} .
\end{aligned}
$$

Thus (B1) holds. Now consider any $e, f \in E$ such that $f \in \omega^{r}(e)$. Using (SBA8), we have that

$$
(f * e) * f=f *(e * f)=f * f=f
$$

and using (SBA7), we have that $f *(f * e)=f * e$. Thus

$$
f \mathcal{R} f * e .
$$

By the dual of (SBA7), we have that $(f * e) * e=f * e$. Using (SBA8), we can see that

$$
e *(f * e)=(e * f) * e=f * e .
$$

Thus $f * e \omega e$. Hence (B21) holds. Now consider any $e, f, g \in E$ such that $g \omega^{l} f$ and $f, g \in \omega^{r}(e)$. By using the dual of (SBA4), we can see that

$$
(g * e) *(f * e)=((g * f) * e) *(f * e)=(g * f) * e=g * e .
$$

Hence (B22) holds. Also, by using (SBA6) we have that

$$
\begin{aligned}
(f * e) *(g * e) & =((e * f) * e) *((e * g) * e) \\
& =((e * f) *(e * g)) * e \\
& =(f * g) * e
\end{aligned}
$$

Hence (B32) holds. Now consider any $e, f, g \in E$ such that $g \omega^{r} f \omega^{r} e$. Using (SBA5), we can see that

$$
(g * e) * f=((e * g) * e) *(e * f)=(e * g) *(e * f)=g * f .
$$

Thus (B31) holds. The duals of (B21), (B22), (B31) and (B32) follow dually. Now to show that (B4) holds, note that by [10, Proposition 2.4] it suffices to show that $(\forall e, f, g \in E)$

$$
\left[f, g \in \omega^{r}(e) \text { and } f * e \omega^{l} g * e\right] \Rightarrow\left(\exists h \in \omega^{r}(e)\right) h \omega^{l} g \text { and } h * e=f * e .
$$


Consider any $e, f, g \in E$ such that $f, g \in \omega^{r}(e)$ and $f * e \omega^{l} g * e$. Let $h=f * g$. Then $h=(e * f) *(e * g)$ so, by (SBA2) and its dual, $h \in \omega^{r}(e) \cap \omega^{l}(g)$. Furthermore, using (SBA6), we have

$$
h * e=((e * f) *(e * g)) * e=((e * f) * e) *((e * g) * e) .
$$

Now, since $f * e \omega^{l} g * e$, that is, since $(e * f) * e \omega^{l}(e * g) * e$, we have that

$$
h * e=(e * f) * e=f * e .
$$

It follows that (B4) holds. The dual of (B4) follows dually. Thus $(E, 0)$ is a biordered set. To show that $(E, \circ)$ is regular it suffices to show that, for all $e, f \in E$, we have that $f * e \in S(e, f)$. Consider any $e, f \in E$. Using (SBA7) and its dual we can easily see that $f * e \in \omega^{l}(e) \cap \omega^{r}(f)$. Now consider any $g \in \omega^{l}(e) \cap \omega^{r}(f)$. Using (SBA8), (SBA5) and then (SBA4), we have that

$$
\begin{aligned}
(e *(f * e)) *(e * g) & =(e * f) *(e * g) \\
& =(e * f) *(e *(f * g)) \\
& =e *(f * g)=e * g .
\end{aligned}
$$

Hence $e * g \omega^{r} e *(f * e)$. Dually, we have that $g * f \omega^{l}(f * e) * f$. Thus

$$
f * e \in S(e, f) \text {. }
$$

Thus $(E, \circ)$ is a regular biordered set. To show that $(E, \circ)$ is solid, consider any $e, f, g \in E$ such that $e \mathcal{L} f \mathcal{R} g$. Then, using (SBA4), we have that

$$
(e * g) * e=(e * g) *(e * f)=(e * g) *(e *(g * f))=e *(g * f)=e
$$

and by using (SBA7), $e *(e * g)=e * g$. Thus $e \mathcal{R} e * g$. Dually, we have that $e * g \mathcal{L} g$. Hence $e * g \in E$ such that

$$
e \mathcal{R} e * g \mathcal{L} g .
$$

It follows that $(E, \circ)$ is a solid regular biordered set. This completes the proof.

Since each solid regular biordered set is the biordered set of a completely regular semigroup, we immediately have

COROLlary 4.9. Each solid binary algebra is determined by a completely regular semigroup.

We now determine which E-solid regular semigroups have the property that their solid binary algebra is a band. (The author thanks Dr. T.E. Hall for pointing out that the converse of the next theorem holds.) 
Theorem 4.10. Consider any $E$-solid regular semigroup $S$ and let $(E, *)$ be the solid binary algebra determined by $S$. Then

$$
(E, *) \text { is a band }
$$

if and only if

$\mathcal{H}$ is a congruence on the core of $S$.

Proof: Let $(E, \circ)$ be the biordered set of idempotents of $S$, let $C$ be the core of $S$ and let $\mu_{C}$ be the maximum idempotent separating congruence on $C$. Since the biordered set of idempotents of $C / \mu_{C}$ is isomorphic to $(E, 0)$, we have that the solid binary algebra determined by $C / \mu_{C}$ is isomorphic to $(E, *)$ (see Section 5). Now suppose that $\mathcal{H}$ is a congruence on $C$. Then since $C$ is completely regular, we have that $C / \mu_{C}$ is a band, and hence is its own solid binary algebra. Therefore $(E, *)$ is a band.

Conversely, suppose that $(E, *)$ is a band. Then, since $(E, 0)$ is the biordered set of the band $(E, *)$, we have that $T_{E}$ is orthodox. Since $C / \mu_{C}$ is idempotent generated and fundamental, we have that (by Result 3.2) $C / \mu_{C}$ is isomorphic to the band of $T_{E}$. Thus $C / \mu_{C}$ is a band and hence $\mathcal{H}_{C}=\mu_{C}$ is a congruence on $C$. This completes the proof.

\section{MORPHISMS, SUBSTRUCTURES AND PRODUCTS}

We now show how morphisms, subalgebras and direct products of solid binary algebras are related to bimorphisms, biordered subsets and direct products of the solid regular biordered sets that determine them.

THEOREM 5.1. Consider any solid regular biordered sets $(E, 0)$ and $(F, 0)$ such that there is a mapping $\theta: E \rightarrow F$. Let $(E, *)$ and $(F, \star)$ be the solid binary algebras determined by $(E, \circ)$ and $(F, \diamond)$, respectively. Then

$$
\theta \text { is a regular bimorphism between }(E, \circ) \text { and }(F, \diamond)
$$

if and only if

$$
\theta \text { is a morphism between }(E, *) \text { and }(F, \star) \text {. }
$$

Proof: Suppose that $\theta$ is a regular bimorphism between $(E, \circ)$ and $(F, \circ)$. Consider any $e, f \in E$ and any $g \in S(e, f)$. By Remark 4.7 (b)(i), we have that

$$
e \circ g \mathcal{R} e * f \mathcal{L} g \circ f
$$


and so, since $\theta$ is a bimorphism, we have

$$
e \theta \diamond g \theta \mathcal{R}(e * f) \theta \mathcal{L} g \theta \diamond f \theta .
$$

Also, since $\theta$ is a regular bimorphism, we have that $g \theta \in S_{F}(e \theta, f \theta)$. Hence, by Remark 4.7 (b)(i) again, we have that

$$
e \theta \diamond g \theta \mathcal{R} e \theta \star f \theta \mathcal{L} g \theta \diamond f \theta .
$$

It now follows easily that $(e * f) \theta=e \theta \star f \theta$. Hence $\theta$ is a morphism between $(E, *)$ and $(F, \star)$.

Conversely, suppose that $\theta$ is a morphism between $(E, *)$ and $(F, \star)$. Then it is easy to show that $\theta$ is a bimorphism between $(E, \circ)$ and $(F, 0)$. To show that $\theta$ is regular, consider any $e, f \in E$. Then by Remark 4.7 (b)(ii) we have that

$$
f * e \in S_{E}(e, f) \text { and }(f * e) \theta=f \theta \star e \theta \in S_{F}(e \theta, f \theta) .
$$

It is now routine to show that, for each $g \in S_{E}(e, f)$, we have

$$
g \theta \in S_{F}(e \theta, f \theta) .
$$

Thus $\theta$ is a regular bimorphism. This completes the proof.

Corollary 5.2. Consider any E-solid regular semigroups $S$ and $T$ such that there is a morphism $\theta: S \rightarrow T$. Then $\theta$ restricted to the set of idempotents of $S$ is a morphism from the solid binary algebra determined by $S$ to the solid binary algebra determined by $T$. Conversely, consider any solid binary algebras $(E, *)$ and $(F, \star)$ such that there is a morphism $\phi: E \rightarrow F$. Then there exist $E$-solid regular semigroups $U$ and $V$ and a morphism $\Phi: U \rightarrow V$ such that

(a) $(E, *)$ and $(F, \star)$ are the solid binary algebras determined by $U$ and $V$, respectively

(b) $\Phi$ restricted to $E$ coincides with $\phi$.

Proof: Follows directly from Theorem 5.1 and the corresponding results for regular biordered sets. See [10, Theorem 1.1 (b3) and Corollary 6.11].

Theorem 5.3. Consider any solid regular biordered sets $(E, \circ)$ and $(F, \diamond)$. Let $(E, *)$ and $(F, \star)$ be the solid binary algebras determined by $(E, \circ)$ and $(F, \diamond)$, respectively. Then

$(F, \diamond)$ is a relatively regular biordered subset of $(E, \circ)$

if and only if

$$
(F, \star) \text { is a subalgebra of }(E, *) .
$$


Proof: Suppose that $(F, \diamond)$ is a relatively regular biordered subset of $(E, \circ)$. Consider any $e, f \in F$ and consider any $g \in S_{F}(e, f)$. By Remark 4.7 (b)(i), we have that

$$
e \circ g \mathcal{R} e \star f \mathcal{L} g \circ f
$$

But, since $(F, \diamond)$ is relatively regular in $(E, \circ)$, it is true that $g \in S_{E}(e, f)$ and hence by Remark 4.7 (b)(i) again, we have that

$$
e \circ g \mathcal{R} e * f \mathcal{L} g \circ f
$$

It is now easy to see that $e * f=e \star f$. Hence $(F, \star)$ is a subalgebra of $(E, *)$.

Conversely, suppose that $(F, \star)$ is a subalgebra of $(E, *)$. Then, since $\star$ is just the restriction of $*$ to $F$, we can easily see that $(F, \diamond)$ is a biordered subset of $(E, \circ)$. By Theorem 5.1, we have that the inclusion mapping $1_{F E}: F \hookrightarrow E$ is a regular bimorphism. Thus $(F, \circ)$ is relatively regular in $(E, \circ)$. This completes the proof.

Corollary 5.4. Consider any $E$-solid regular semigroup $S$ and let $(E, *)$ be the solid binary algebra determined by $S$. Consider any subset $F$ of $E$. Then

the subsemigroup generated by $F$ is a regular subsemigroup $R$ of $S$ such that $F$ is the set of idempotents of $R$

if and only if

$F$ is the underlying set of a subalgebra of $(E, *)$.

Proof: Follows directly from Theorem 5.3 and the corresponding result for regular biordered sets. See [10, Theorem 1.3].

Theorem 5.5. Consider any family $\left\{\left(E_{i}, \circ_{i}\right) \mid i \in I\right\}$ of solid regular biordered sets and let $(E, \circ)=\prod_{i \in I}\left(E_{i}, \circ_{i}\right)$. For each $i \in I$, let $\left(E_{i}, *_{i}\right)$ be the solid binary algebra determined by $\left(E_{i}, \circ_{i}\right)$. Finally, let $(E, *)=\prod_{i \in I}\left(E_{i}, *_{i}\right)$. Then $(E, \circ)$ is the solid regular biordered set that determines $(E, *)$.

Proof: By Result 3.6, we have that $(E, \circ)$ is a regular biordered set and it is easy to verify that $(E, 0)$ is solid. Furthermore, one can easily show that $*$ satisfies properties (a) and (b) of Theorem 4.6. Hence $(E, \circ)$ determines $(E, *)$. This completes the proof.

Corollary 5.6. Consider any family $\left\{S_{i} \mid i \in I\right\}$ of E-solid regular semigroups and let $\left(E_{i}, *_{i}\right)$ be the solid binary algebra determined by $S_{i}$, for each $i \in I$. Then $\prod_{i \in I}\left(E_{i}, *_{i}\right)$ is the solid binary algebra determined by $\prod_{i \in I} S_{i}$.

Proof: Follows directly from Theorem 5.5 and Result 3.7. 


\section{Solid LOCAL SEMILATTICES}

The following definition is due to Nambooripad [11].

Definition 6.1: (Nambooripad) Consider any nonempty set $E$ and any binary operation $\wedge: E \times E \rightarrow E$. Define $(E, \wedge)$ to be a local semilattice algebra if and only if $(E, \wedge)$ satisfies the following identities and their duals:

(LSL1) $x \wedge x=x$

(LSL2) $\quad(x \wedge y) \wedge(x \wedge z)=(x \wedge y) \wedge z$

(LSL3) $\quad((x \wedge u) \wedge(x \wedge v)) \wedge(x \wedge w)=(x \wedge u) \wedge((x \wedge v) \wedge(x \wedge w))$.

Nambooripad [11] has shown that, for each local semilattice $(E, 0)$, there is a unique binary operation $\wedge: E \times E \rightarrow E$ such that

(a) $(E, \wedge)$ is a local semilattice algebra

(b) $\circ$ is $\wedge$ restricted to the domain

$D_{E}^{\wedge}=\{(e, f) \in E \times E \mid e \wedge f=e, e \wedge f=f, f \wedge e=f$ or $f \wedge e=e\}$

we shall call the resulting algebra $(E, \wedge)$ the local semilattice algebra determined by $(E, 0)$. Furthermore, he showed that

$$
(\forall e, f \in E)\{. \quad \cdot \quad f, e) .
$$

Conversely, Nambooripad showed that every local semilattice algebra $(E, \wedge)$ is determined by the local semilattice $\left(E, \wedge \mid D_{E}^{\wedge}\right)$.

Now if $(E, \circ)$ is a solid local semilattice then we can extend $\circ$ to $*$ so that $(E, *)$ is a solid binary algebra and we can extend $\circ$ to $\wedge$ so that $(E, \wedge)$ is a local semilattice algebra. By Remark 4.7 (b)(ii), we have that

$$
(\forall e, f \in E) e * f \in S(f, e)
$$

and so $*=\wedge$. Furthermore, the next theorem shows us that $(E, *)$ is a normal band.

THEOREM 6.2. An algebra which is both a local semilattice algebra and a solid binary algebra is a normal band.

Proof: Consider any algebra $E$ which is both a local semilattice algebra and a solid binary algebra (multiplication will be written as juxtaposition of elements). To show that $E$ is a normal band it suffices to show that $E$ satisfies the following identities

(i) $(a b) c=a(b c)$

(ii) $a b c a=a c b a$. 
Consider any $a, b, c \in E$. By identity (SBA4), we have that $a(b c)=(a b)(a(b c))$ and by identity (LSL2), we have that $(a b)(a(b c))=(a b)(b c)$. Hence

$$
a(b c)=(a b)(b c) \text {. }
$$

Dually, it is true that $(c b) a=(c b)(b a)$ and hence (by interchanging $a$ and $c$ ), we have that

$$
(a b) c=(a b)(b c) .
$$

Thus $(a b) c=(a b)(b c)=a(b c)$. We now know that $E$ is a semigroup and so multiplication of elements may be written unambiguously without brackets. To show that the second identity holds, note that since every element of $E$ is idempotent, we have that

$$
a b c a=a b c b c a .
$$

Furthermore, by identity (LSL2) and its dual, we have that

$$
a b c b c a=a b c b a=a c b a .
$$

Thus $a b c a=a c b a$. Hence $\dot{E}$ is a normal band, as required.

We immediately have the following (probably well-known) stronger version of $[\mathbf{1 0}$, Theorem 7.7]:

Corollary 6.3. Consider any biordered set $(E, \circ)$. Then the following are equivalent:

(a) $(E, o)$ is a solid local semilattice

(b) $(E, \circ)$ is the biordered set of a normal band.

\section{REFERENCES}

[1] R. Broeksteeg, 'The structure of solid binary algebras', (preprint).

[2] A.H. Clifford and G.B. Preston, The algebraic theory of semigroups, Math. Surveys No.7 (American Mathematical Society, Providence, R.I., 1961) (reprinted 1988).

[3] D. Easdown, 'Biordered sets come from semigroups', J. Algebra 96 (1985), 581-591.

[4] T.E. Hall, 'On regular semigroups', J. Algebra 24 (1973), 1-24.

[5] P.M. Higgins, Techniques of semigroup theory (Oxford University Press, 1992).

[6] J.M. Howie, An introduction to semigroup theory, London Math. Soc. Monographs 7 (Academic Press, London, New York, 1976).

[7] J. Meakin, 'Local semilattices on two generators', Semigroup Forum 24 (1982), 95-116.

[8] J. Meakin and F. Pastijn, 'The free pseudo-semilattice on two generators', Algebra Universalis 14 (1982), 297-309. 
[9] K.S.S. Nambooripad, 'Structure of regular semigroups I: Fundamental regular semigroups', Semigroup Forum 9 (1975), 354-363.

[10] K.S.S. Nambooripad, 'Structure of regular semigroups I', Mem. Amer. Math. Soc. 22 (1979 224).

[11] K.S.S. Nambooripad, 'Pseudo-semilattices and biordered sets I', Simon Stevin 55 (1981), 103-110.

[12] Y.T. Yeh, 'The existence of e-free objects in e-varieties of regular semigroups', Internat. J. Algebra Comput. 2 (1992), 471-484.

Mathematics Department

Monash University

Clayton Vic 3168

Australia

e-mail: apm485g@vaxc.cc.monash.edu.au 